\title{
Veratramine suppresses human HepG2 liver cancer cell growth in vitro and in vivo by inducing autophagic cell death
}

\author{
LINLIN YIN $^{1 *}$, YONGHUI XIA ${ }^{2 *}$, PING XU $^{1}$, WENLI ZHENG ${ }^{1}$, \\ YUANYUAN GAO $^{1}$, FAQIN XIE ${ }^{1}$ and ZHAONING JI ${ }^{1}$ \\ ${ }^{1}$ Department of Oncology, Yijishan Hospital of Wannan Medical College; ${ }^{2}$ Department of Respiratory Medicine, \\ The Second Affiliated Hospital of Wannan Medical College, Wuhu, Anhui 241001, P.R. China
}

Received September 22, 2019; Accepted April 7, 2020

DOI: $10.3892 /$ or.2020.7622

\begin{abstract}
Liver cancer is the second leading cause of cancer-related deaths. Traditional therapeutic strategies, such as chemotherapy, targeted therapy and interventional therapy, are inefficient and are accompanied by severe side effects for patients with advanced liver cancer. Therefore, it is crucial to develop a safer more effective drug to treat liver cancer. Veratramine, a known natural steroidal alkaloid derived from plants of the lily family, exerts anticancer activity in vitro. However, the underlying mechanism and whether it has an antitumor effect in vivo remain unknown. In the present study, the data revealed that veratramine significantly inhibited HepG 2 cell proliferation, migration and invasion in vitro. Moreover, it was revealed that veratramine induced autophagy-mediated apoptosis by inhibiting the PI3K/Akt/ mTOR signaling pathway, which partly explained the underlying mechanism behind its antitumor activity. Notably, the results of in vivo experiments also revealed that veratramine treatment $(2 \mathrm{mg} / \mathrm{kg}, 3$ times a week for 4 weeks) significantly inhibited subcutaneous tumor growth of liver cancer cells, with a low systemic toxicity. Collectively, the results of the present study indicated that veratramine efficiently suppressed liver cancer HepG2 cell growth in vitro and in vivo by blocking the $\mathrm{PI} 3 \mathrm{~K} / \mathrm{Akt} / \mathrm{mTOR}$ signaling pathway to induce autophagic cell death. Veratramine could be a potential therapeutic agent for the treatment of liver cancer.
\end{abstract}

Correspondence to: Professor Faqin Xie or Professor Zhaoning Ji, Department of Oncology, Yijishan Hospital of Wannan Medical College, 2 West Zheshan Road, Wuhu, Anhui 241001, P.R. China

E-mail: xiefq33@163.com

E-mail: yjsjzning@163.com

*Contributed equally

Key words: veratramine, liver cancer, autophagic cell death, apoptosis, PI3K/Akt/mTOR pathway

\section{Introduction}

Liver cancer is one of the most common malignant tumor types, with an incidence rate ranked sixth, and a mortality rate ranked third worldwide (1). Liver cancer is characterized by a rapid development, poor curative effect and high recurrence rate, and the five-year survival rate is $<30 \%(2,3)$. Surgery is the only available method to treat developed cases, but most patients are diagnosed at an advanced stage and have already lost the opportunity for surgery. At present, drug treatment including chemotherapy and targeted therapy is not effective for the treatment of liver cancer (3-5), and the prognosis of liver cancer patients is still poor $(4,6,7)$. Therefore, it is crucial to develop a more efficient and safer drug for the treatment of liver cancer.

Autophagy, a highly conserved biological process in eukaryotic cells, is a lysosomal-mediated metabolic process that degrades damaged proteins or organelles to maintain cell metabolism and cell renewal, which plays a key role in maintaining homeostasis in the cellular environment (8-10). Dysregulation of autophagy is associated with a variety of diseases including multiple forms of cancer $(11,12)$. Autophagy plays a 'double-edged sword' role, promotion and suppression, in tumor cells, which depends on the type and stage of tumor development (13). In the early stages of cancer cell expansion, autophagy provides energy and nutrients to normal cells, which can inhibit the proliferation of cancer cells (14). During tumor progression, autophagy can enhance the tolerance of cancer cells to low-nutrient environments to promote cancer cell proliferation, and also increase the resistance of cancer cells to anticancer drugs (15). A previous study has revealed that deletion of the autophagy marker gene ATG6/Beclin-1 often occurs in a variety of cancer types (16). In addition, in animal models, Beclin-1 gene-deficient mice were more susceptible to developing cancer types (17). The aforementioned studies indicated that Beclin-1 is a tumor suppressor gene, and that autophagy is closely related to the occurrence and development of tumors. Generally, autophagy is a non-specific degradation of intracellular components, but autophagy can also specifically degrade some target proteins $(9,10)$. If specific cytoprotective factors are degraded, autophagic cell death will occur (18). Autophagy and apoptosis, two different forms of programmed cell death, often occur simultaneously when 
cells are under stress (19). The relationship between autophagy and apoptosis is a research hotspot. Studies have revealed that there are four relationships between autophagy and apoptosis $(19,20)$ : i) Autophagy precedes apoptosis and maintains cell homeostasis; ii) apoptosis inhibits autophagic cell death and inhibition of apoptosis stimulates autophagic cell death; iii) autophagy degrades damaged organelles and proteins as well as attenuating DNA damage, thereby inhibiting apoptosis; iv) autophagy and apoptosis exist independently. The gene regulatory network behind autophagy and apoptosis is highly linked. The anti-apoptotic protein B-cell lymphoma 2 (Bcl-2) inhibits the formation of autophagosomes by binding to the pro-autophagic protein, Beclin-1 $(21,22)$. Notably, caspases, a highly studied group of essential apoptotic proteins, can cause the inhibition of autophagy by breaking down autophagy-associated proteins, including p62, Beclin-1, ATG5 and ATG3 (23-25). In addition, multiple autophagy-related proteins are also involved in apoptosis when cells are under stress $(26,27)$. Mammalian target of rapamycin (mTOR), a serine/threonine protein kinase, is involved in various physiological functions, such as cell proliferation, apoptosis, autophagy and the cell cycle $(28,29)$. mTOR is primarily activated by the PI3K/AKT/mTOR signaling pathway (28). The activated PI3K/AKT/mTOR signaling pathway plays an important role in the occurrence and development of various tumor types (30). Inhibition of this pathway has become an important target for cancer therapy, and multiple mTOR inhibitors have shown satisfactory efficacy (31-33).

Veratramine, a known natural steroidal alkaloid, is found in a variety of plants of the lily family. Previous studies have revealed that veratramine lowers blood pressure, antagonizes sodium ion channels and functions as an analgesic. A recent study revealed that veratramine inhibited tumor cell proliferation in vitro (34). Bai et al (35) also revealed that veratramine inhibited the downstream signaling pathway of transcription factor activator protein-1 (AP-1) that regulates multiple cell functions including proliferation, apoptosis and epithelial-mesenchymal transition (EMT). These results indicated that veratramine may have antitumor activity, but the effect of veratramine on liver cancer and the mechanism behind its antitumor activity are unclear. In the present study, the aim was to investigate the effects of veratramine on liver cancer in vitro and in vivo. In addition, as part of the present work, a focus was applied to investigate whether veratramine can induce autophagic cell death in HepG2 cells, in order to clarify the method of action behind its antitumor activity.

\section{Materials and methods}

Cell lines and reagents. Veratramine (with a purity $\geq 98 \%$ ) was extracted and isolated from the roots and rhizomes of Veratrum nigrum L., and provided by Nanjing Spring \& Autumn Biological Engineering Co., Ltd. The chemical structure of veratramine is presented in Fig. 1A. The human liver cancer HepG2 cell line was purchased from the American Type Culture Collection. FBS, penicillin streptomycin and cell culture media were purchased from Hangzhou Sijiqing Biological Engineering Materials Co., Ltd. The Cell Counting Kit-8 (CCK-8) assay kit was obtained from Nanjing KeyGen Biotech Co., Ltd. The apoptosis detection kit and antibodies used for western blotting were purchased from Beyotime Institute of Biotechnology. The reverse transcription kit and PCR kit were purchased from Vazyme Biotech Co., Ltd. Acridine orange (AO) and 3-methyladenine (3-MA) were obtained from Sigma-Aldrich; Merck KGaA. All other reagents and solvents (AR grade) were purchased from Sinopharm Chemical Reagent Co., Ltd. Ten BALB/c nude mice, aged 4-6 weeks (16-18 g), were obtained from Shanghai Slack Laboratory Animals Co., Ltd. These mice were housed in specialized mouse cages at $\sim 20^{\circ} \mathrm{C}$, humidity $50-60 \%$, specific pathogen-free, dark conditions, and fed with normal food and clean water.

Cell culture. The human liver cancer HepG2 cells were cultured in RPMI-1640 media containing 10\% FBS, penicillin (100 units/ml) and streptomycin (100 units/ml). The cells were maintained in a humid incubator (Sanyo XD-101; Sanyo) with $5 \% \mathrm{CO}_{2}$ at $37^{\circ} \mathrm{C}$.

Cell viability assay. Cell viability was assessed using a CCK-8 assay. HepG 2 cells $\left(5 \times 10^{3}\right.$ cells/well) were seeded in 96 well plates for $24 \mathrm{~h}$, and then treated with various concentrations of veratramine $(0,2.5,5,10,20,40$ and $80 \mu \mathrm{M})$ for an additional 24,48 or $72 \mathrm{~h}$. Subsequently, $10 \mu \mathrm{l}$ of CCK-8 solution was added to each well and the cells were further incubated for $4 \mathrm{~h}$. The absorbance was measured at $450 \mathrm{~nm}$ using a microplate reader (Bio-Rad Laboratories, Inc.). $\mathrm{IC}_{50}$ values were calculated using the cell survival data curve.

Cell migration and invasion assay. The effect of veratramine on HepG2 cell migration and invasion was assessed using a wound healing assay and Transwell assay, respectively. For the wound healing assay, HepG2 cells were seeded in 6 well plates and cultured to $90 \%$ confluence. The cell monolayer was scratched using a $10-\mu 1$ pipette tip. Subsequently, the cells were treated with veratramine $(40 \mu \mathrm{M})$ for $48 \mathrm{~h}$. After drug treatment, the cells were observed through an inverted microscope (Olympus Corporation), and the width of the scratch was measured.

For the Transwell assay, Transwell culture inserts were placed into the wells of 6-well plates and coated with a layer of Matrigel $^{\mathrm{TM}}$. Subsequently, RPMI-1640 medium containing $10 \%$ FBS was added to the lower chamber and HepG2 cells were seeded in the upper chamber. After veratramine $(40 \mu \mathrm{M})$ treatment for $48 \mathrm{~h}$, the invaded cells in the lower chamber were fixed with $1 \%$ formaldehyde for $10 \mathrm{~min}$ at $25^{\circ} \mathrm{C}$ and stained with $0.5 \%$ crystal violet for another $5 \mathrm{~min}$. Finally, the cells were observed under an inverted microscope (magnification, $\mathrm{x} 200$ ).

Detection of apoptosis. Cell apoptosis was evaluated using the Annexin V-FITC/7AAD double staining assay. Briefly, HepG2 cells $\left(3 \times 10^{5}\right.$ cells/well) were seeded in 6 -well plates for $24 \mathrm{~h}$. Subsequently, the cells were treated with increasing concentrations of veratramine $(10,20$ and $40 \mu \mathrm{M})$ for $48 \mathrm{~h}$. After incubation, the cells were harvested and stained with Annexin V-FITC and 7AAD according to the manufacturer's instructions. Apoptotic cells were quantified using flow cytometry (FACSCalibur; BD Biosciences) and the data were analyzed using FlowJo software (FlowJo V10; Tree Star, Inc.). 

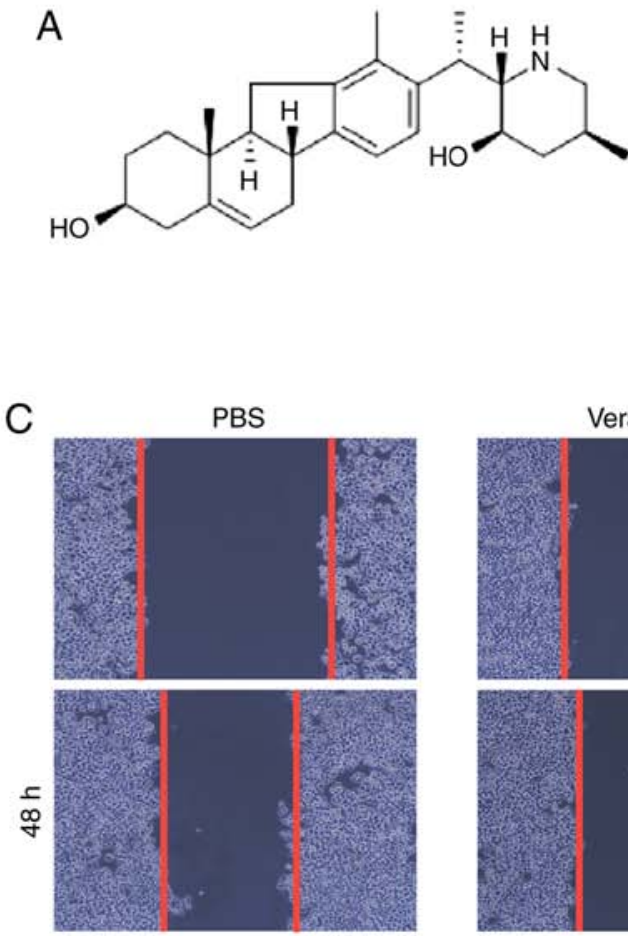

E

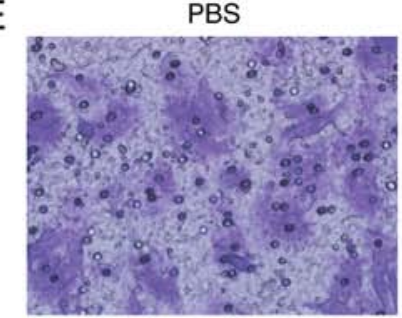

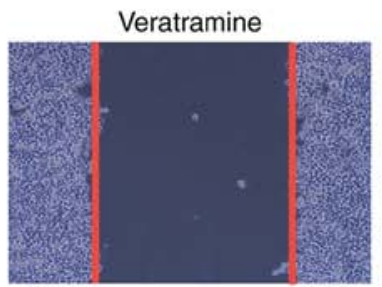
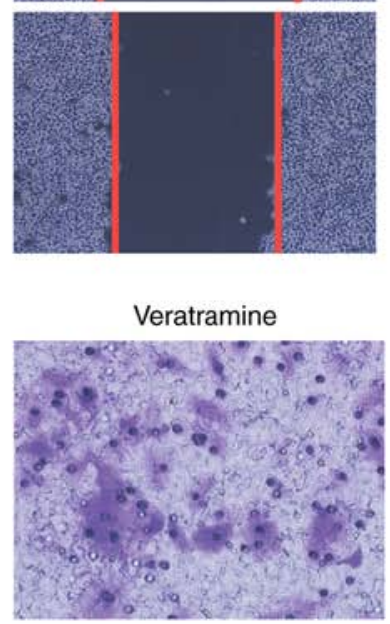
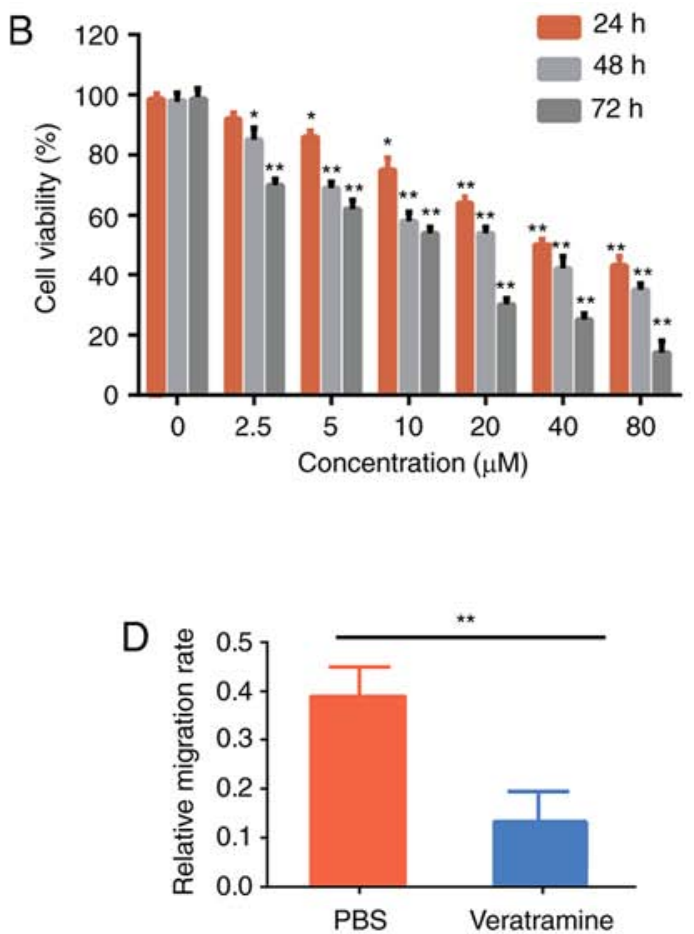

$\mathrm{F}$

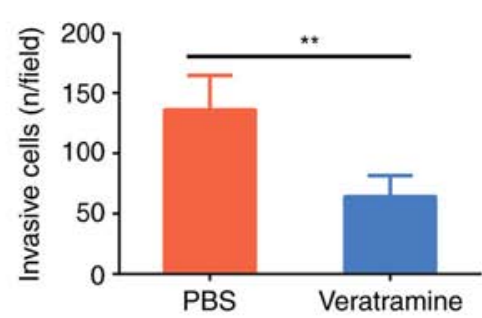

Figure 1. Veratramine inhibits HepG2 cell proliferation, migration and invasion in vitro. (A) Structure of veratramine. (B) HepG2 cells were treated with various concentrations of veratramine $(0,2.5,5,10,20,40$ and $80 \mu \mathrm{M})$ for 24,48 and $72 \mathrm{~h}$. The cell viability was determined using CCK-8 assays. (C) HepG2 cells were treated with veratramine $(40 \mu \mathrm{M})$ for $48 \mathrm{~h}$. Cell migration was analyzed using wound scratch assays. Representative images are presented (magnification, x200). (D) Statistical data for the wound scratch assays. (E) Cells were treated as described and cell invasion was detected using Transwell assays. Representative images are presented (magnification, x200). (F) Quantitative analysis of invading cells. Data are presented as the mean \pm SD of three independent experiments. ${ }^{*} \mathrm{P}<0.05,{ }^{* * *} \mathrm{P}<0.01$ vs. the control group (PBS).

Total RNA isolation and reverse transcription-quantitative $P C R(R T-q P C R)$. RT-qPCR was used to evaluate the expression levels of the key apoptotic genes, BCL2 and BAX, and $A P-1$ downstream gene ETS1. After phosphate-buffered saline (PBS) or veratramine treatment for $48 \mathrm{~h}$, HepG 2 cells were subjected to total RNA isolation using TRIzol ${ }^{\circledR}$ reagent. Subsequently, cDNA was synthesized using the HiScript III 1st Strand cDNA Synthesis kit and qPCR was performed using the ChamQ SYBR qPCR Master Mix according to the manufacturer's instructions. Thermocycling parameters were optimized as $5 \mathrm{~min}$ at $95^{\circ} \mathrm{C}$, followed by 40 cycles at $95^{\circ} \mathrm{C}$ $(15 \mathrm{sec}), 60^{\circ} \mathrm{C}(30 \mathrm{sec})$; melt curve: $95^{\circ} \mathrm{C}(15 \mathrm{sec}), 60^{\circ} \mathrm{C}(30 \mathrm{sec})$ and $95^{\circ} \mathrm{C}(15 \mathrm{sec})$. PCR primers were provided by Guangzhou RiboBio Co., Ltd., and $\beta$-actin was used as the internal reference. Quantitative analysis of relative mRNA expression levels was performed according to the $2^{-\Delta \Delta \mathrm{Cq}}$ method (36). Primer sequences were as follows: BCL2 forward, 5'GTGGAGGAG
CTCTTCAGGGA3' and reverse, 5'AGGCACCCAGGGTGA TGCAA3'); BAX forward, 5'GGCCCACCAGCTCTGAGC AGA3' and reverse, 5'GCCACGTGGGCGTCCCAAAGT3'; ETS1 forward, 5'GCGCGCTAGCAACTTGCTACCATC CCGT3' and reverse, 5'GCGCAAGCTTTGCCATCACTC GTCGGC3'; $\beta$-actin forward, 5'ACAAAGTGGTCATTG AGGGC3'and reverse, 5'GCCGTCAGGCAGCTCGTAGC3'.

Acridine orange staining. Formation of acidic vesicular organelles (AVO) is a hallmark of autophagic cells. Acridine orange (AO) dye readily penetrates cell membranes and visualizes the occurrence of the acidic vesicular organelles (AVOs). Therefore, AO staining was used to detect autophagy. HepG2 cells were cultured overnight and treated with varying concentrations of veratramine $(10,20$ and $40 \mu \mathrm{M})$ for $48 \mathrm{~h}$. Subsequently, the cells were stained with $10 \mu \mathrm{g} / \mathrm{ml}$ of $\mathrm{AO}$ dye for $20 \mathrm{~min}$ at $37^{\circ} \mathrm{C}$ in the dark. AVO formation (Red) 
A

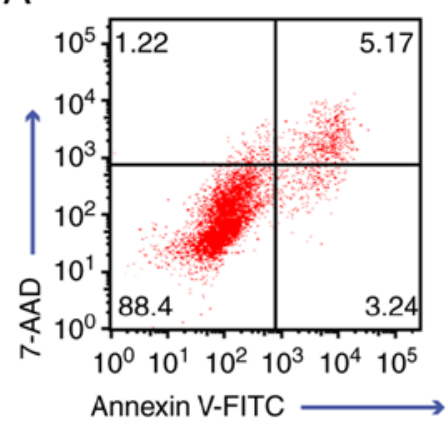

$10 \mu \mathrm{M}$

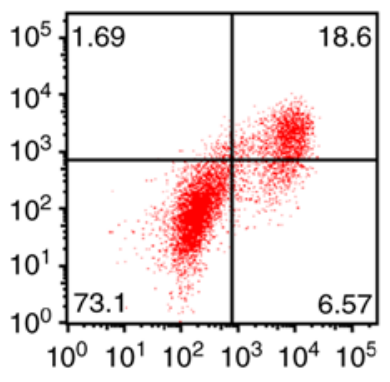

$20 \mu \mathrm{M}$

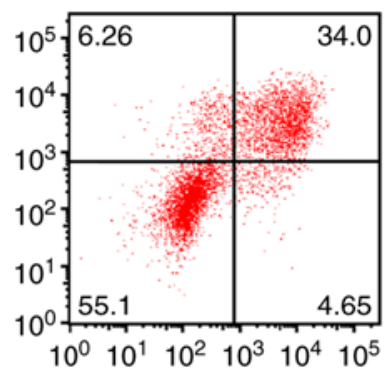

$40 \mu \mathrm{M}$

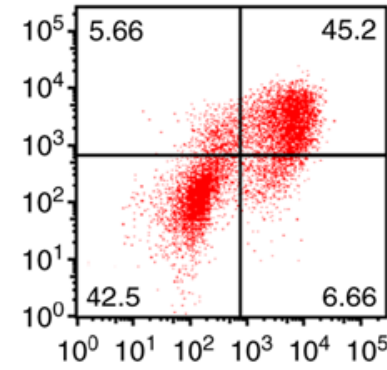

B

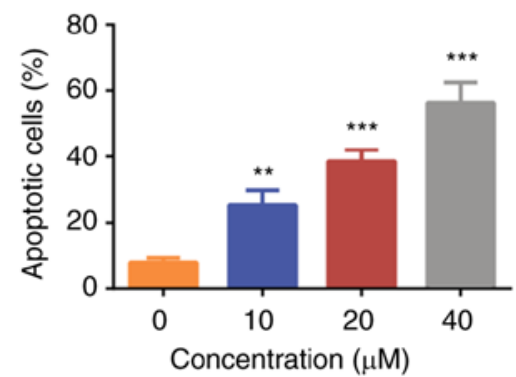

C

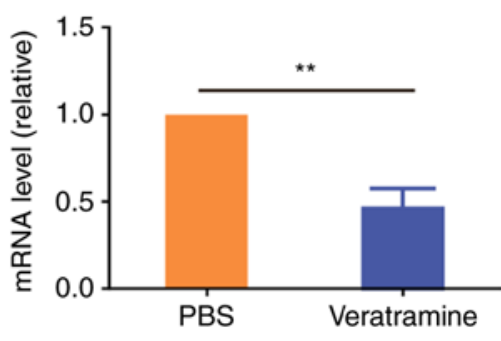

$B A X$

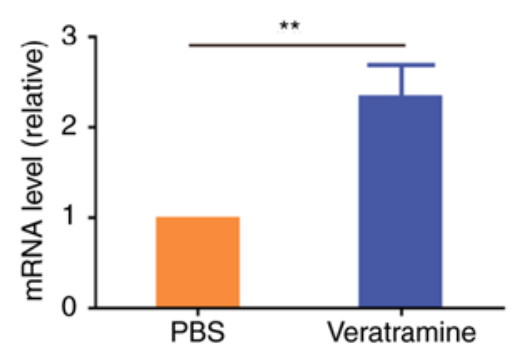

Figure 2. Veratramine induces HepG2 cell apoptosis in a dose-dependent manner. (A) HepG2 cells were treated with various concentrations of veratramine $(0,10,20$ and $40 \mu \mathrm{M})$ for $48 \mathrm{~h}$. Cells were treated with Annexin V-FITC/7AAD double staining for flow cytometric analysis. Representative images are presented. (B) Statistical data of the apoptotic cells (Annexin $\mathrm{V}^{+}$). (C) Quantitative RT-qPCR analysis of the anti-apoptotic gene, BCL2, and (D) the pro-apoptotic gene, $B A X$, expression levels in HepG2 cells treated with PBS or veratramine $(40 \mu \mathrm{M})$ for $48 \mathrm{~h}$. The data are presented as the mean \pm SD of three independent experiments. ${ }^{* *} \mathrm{P}<0.01,{ }^{* * *} \mathrm{P}<0.001$ vs. the control group (PBS). BCL2, B-cell lymphoma 2; BAX, Bcl-2 associated X protein.

was observed using fluorescence microscopy (magnification, $\mathrm{x} 200$ ). The percentage of autophagic cells was measured using flow cytometry.

Western blotting. HepG 2 cells were cultured overnight and then treated with varying concentrations of veratramine $(10,20$ and $40 \mu \mathrm{M}$ ) for $48 \mathrm{~h}$. An equal volume of PBS was used as a control. After drug treatment, the cells were harvested and lysed in RIPA lysis buffer supplemented with a protease inhibitor cocktail. Cell debris were removed by centrifugation at $14,000 \times$ g at $4{ }^{\circ} \mathrm{C}$ for $15 \mathrm{~min}$ and the protein concentration was determined using BCA assays. Subsequently, $30 \mu \mathrm{g}$ of total protein was separated on a $10 \%$ SDS-PAGE gel and electroblotted onto PVDF membranes (EMD Millipore). The membranes were blocked with $5 \%$ skim milk in TBS-Tween-20 (TBST; $20 \mathrm{mmol} / 1$ Tris-HCl, $150 \mathrm{mmol} / 1 \mathrm{NaCl}$ and $0.1 \%$ Tween-20) for $1 \mathrm{~h}$ at room temperature (RT), and then incubated with anti-Beclin1 (product no. 3495), anti-LC3 (product no. 3868), anti-PI3K (product no. 4249), anti-phospho-PI3K (product no. 17366), anti-Akt (product no. dilution 4685), anti-phospho-Akt (product no. 9614), anti-mTOR (product no. 2983), anti-phospho-mTOR (product no. 5536) or anti-GAPDH (product no. 5174; all with a dilution 1:1,000; all from Cell Signaling Technology, Inc.) primary antibodies at $4^{\circ} \mathrm{C}$, overnight. After washing $3 \times 10$ min with TBST, the membranes were incubated with horseradish peroxidase-conjugated secondary antibodies (product no. 7074; dilution 1:2,000; Cell Signaling Technology, Inc.) for $1 \mathrm{~h}$ at RT. The protein bands were visualized using ECL reagent and the G:BOX chemiXR5 Imaging System (Syngene). Band intensities were quantified using Image-Pro Plus 6.0 software, with GAPDH used as an internal control.

Anti-tumor growth effects in vivo. The liver cancer xenograft model was established by subcutaneous injection of human HepG2 cells $\left(1 \times 10^{6}\right.$ cells suspended in $0.1 \mathrm{ml} \mathrm{PBS}$ for each mouse) into the armpits of nude female BALB/c mice (6-8 weeks old). Once the tumors reached a volume of approximately $75-100 \mathrm{~mm}^{3}$, the mice were randomly divided into two groups, with five mice per group, a PBS group and veratramine group. PBS $(200 \mu \mathrm{l})$ or veratramine $(2 \mathrm{mg} / \mathrm{kg})$ were injected into the mice via the tail vein. Based on the $\mathrm{IC}_{50}$ value of veratramine on HepG2 cells in vitro, and previous studies (37-39), $2 \mathrm{mg} / \mathrm{kg}$ of veratramine was used for the animal experiment. The mice were treated three times a week for 4 weeks. During the treatment, the weights of the mice and the volume of the tumors were measured every week. The volume of the tumors was calculated according to the following formula: $\mathrm{V}=0.5 \mathrm{x}$ length $\mathrm{x}$ width $^{2}$. Three days after the last treatment, blood was collected from the mice and the mice were sacrificed. Subcutaneous tumors from the mice were excised and weighed. The number of white blood cells, neutrophils and platelets, as well as the concentrations of alanine aminotransferase, aspartate aminotransferase and creatinine were examined. The heart, liver, spleen, lungs and kidneys were excised and then fixed with $4 \%$ paraformaldehyde. The paraffin sections of the organs were performed for pathological evaluation with hematoxylin-eosin (H\&E) staining (hematoxylin, $5 \mathrm{~min}$; eosin, $2 \mathrm{~min}$ ) at room temperature. The results of $H \& E$ staining were evaluated by pathologist in a 
A

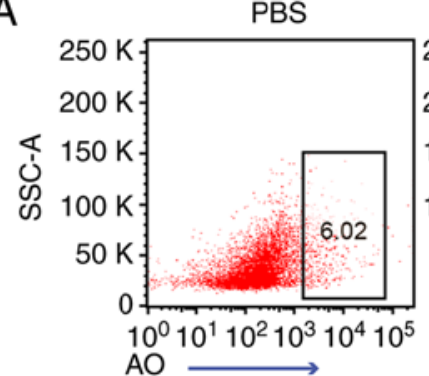

B

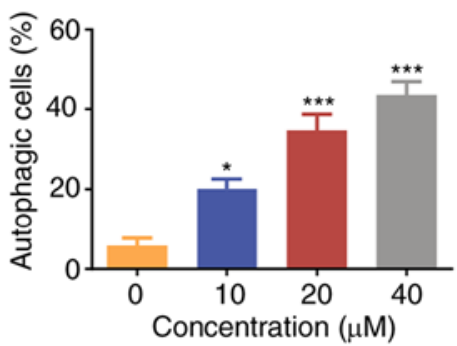

D

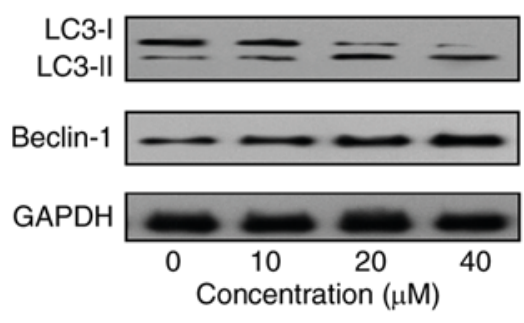

$10 \mu \mathrm{m}$

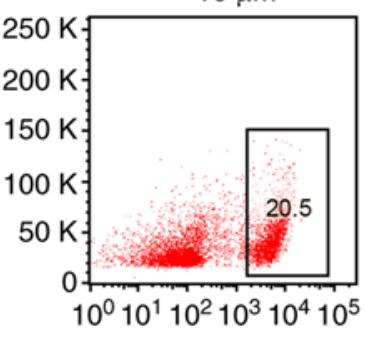

C

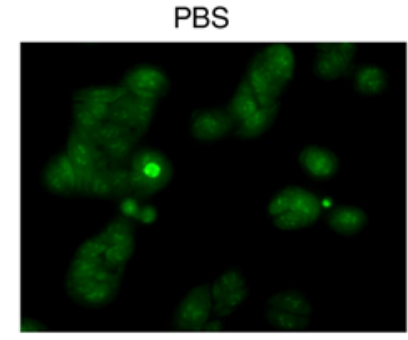

$20 \mu \mathrm{m}$

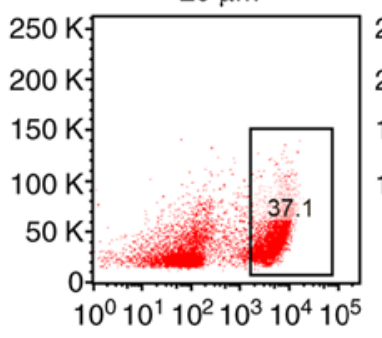

$40 \mu \mathrm{m}$

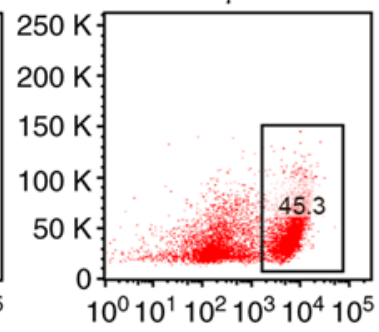

Veratramine $(40 \mu \mathrm{M})$

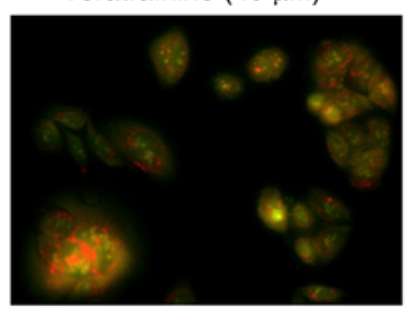

F

E

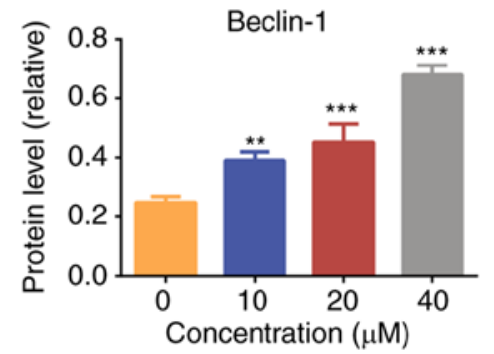

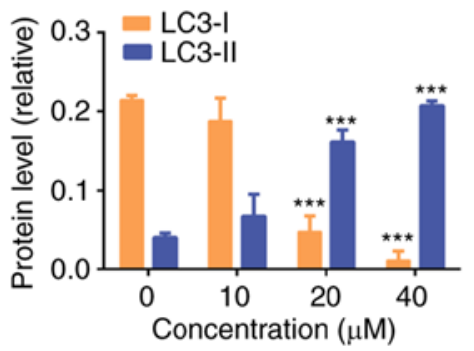

Figure 3. Veratramine induces HepG2 cell autophagy in a dose-dependent manner. (A) Flow cytometric analysis of autophagic vacuoles (AO ${ }^{+}$) in HepG2 cells treated with various concentrations of veratramine $(0,10,20$ and $40 \mu \mathrm{M})$ for $48 \mathrm{~h}$. (B) Percentages of the autophagic cells. (C) Representative fluorescence microscopy images of autophagic vacuoles (red) in HepG2 cells treated with PBS or veratramine (40 $\mu \mathrm{M}$ ) for $48 \mathrm{~h}$. (D) Western blot analysis of the autophagy marker proteins, Beclin-1 and LC3, in HepG2 cells after treatment. Quantitative analysis of (E) Beclin-1 as well as (F) LC3-I and LC3-II. The data are presented as the mean $\pm \mathrm{SD}$ of three independent experiments. ${ }^{*} \mathrm{P}<0.05,{ }^{* *} \mathrm{P}<0.01,{ }^{* * *} \mathrm{P}<0.001$ vs. the control group (PBS).

blinded fashion. The animal experiments were approved by the Animal Care and Use Committee of Yijishan Hospital of Wannan Medical College.

Statistical analysis. The experiments were performed in triplicate and the data are presented as the mean \pm SD. Data were analyzed using GraphPad Prism 6.0 software (GraphPad Software, Inc.). Student t-tests or ANOVAs with Tukey's post hoc test were used to evaluate the data, and $\mathrm{P}<0.05$ was considered to indicate a statistically significant difference.

\section{Results}

Veratramine inhibits HepG2 cell proliferation, migration and invasion. CCK-8, wound healing and Transwell assays were performed to examine the effects of veratramine on HepG2 cell proliferation, migration and invasion, respectively. As revealed in Fig. 1B, veratramine significantly inhibited HepG2 cell proliferation in a time- and dose-dependent manner, and the $\mathrm{IC}_{50}$ values of veratramine on HepG2 cells for 24,48 , and $72 \mathrm{~h}$ were $26.54 \mu \mathrm{M}, 19.81$ and $9.12 \mu \mathrm{M}$, respectively.
Furthermore, the data revealed that $40 \mu \mathrm{M}$ of veratramine treatment reduced the cell migration rate compared to the PBS control (Fig. 1C and D). Consistently, the number of invasive cells was also decreased after veratramine treatment for $48 \mathrm{~h}$. Collectively, these results indicated that veratramine not only suppressed cell growth but also reduced cell migration and invasion in HepG2 cells. This demonstrated the potential for veratramine to treat liver cancer.

Veratramine induces apoptosis in HepG2 cells. The effect of veratramine on apoptosis was investigated using Annexin V-FITC/7AAD double staining and RT-qPCR. The cells were treated with the indicated dose of veratramine for $48 \mathrm{~h}$ and the same volume of PBS was used as a negative control. As indicated in Fig. 2A and B, flow cytometric data revealed that veratramine induced apoptosis in HepG2 cells, and the proportion of apoptotic cells (Annexin $\mathrm{V}^{+}$) increased from $8.41 \%$ (PBS control) to $51.86 \%$ (40 $\mu \mathrm{M}$ of veratramine). Although multiple signaling pathways are involved in apoptosis, the majority of them are regulated by the Bcl-2 family (40). $B C L 2$ and $B A X$ are representative genes in the 
A
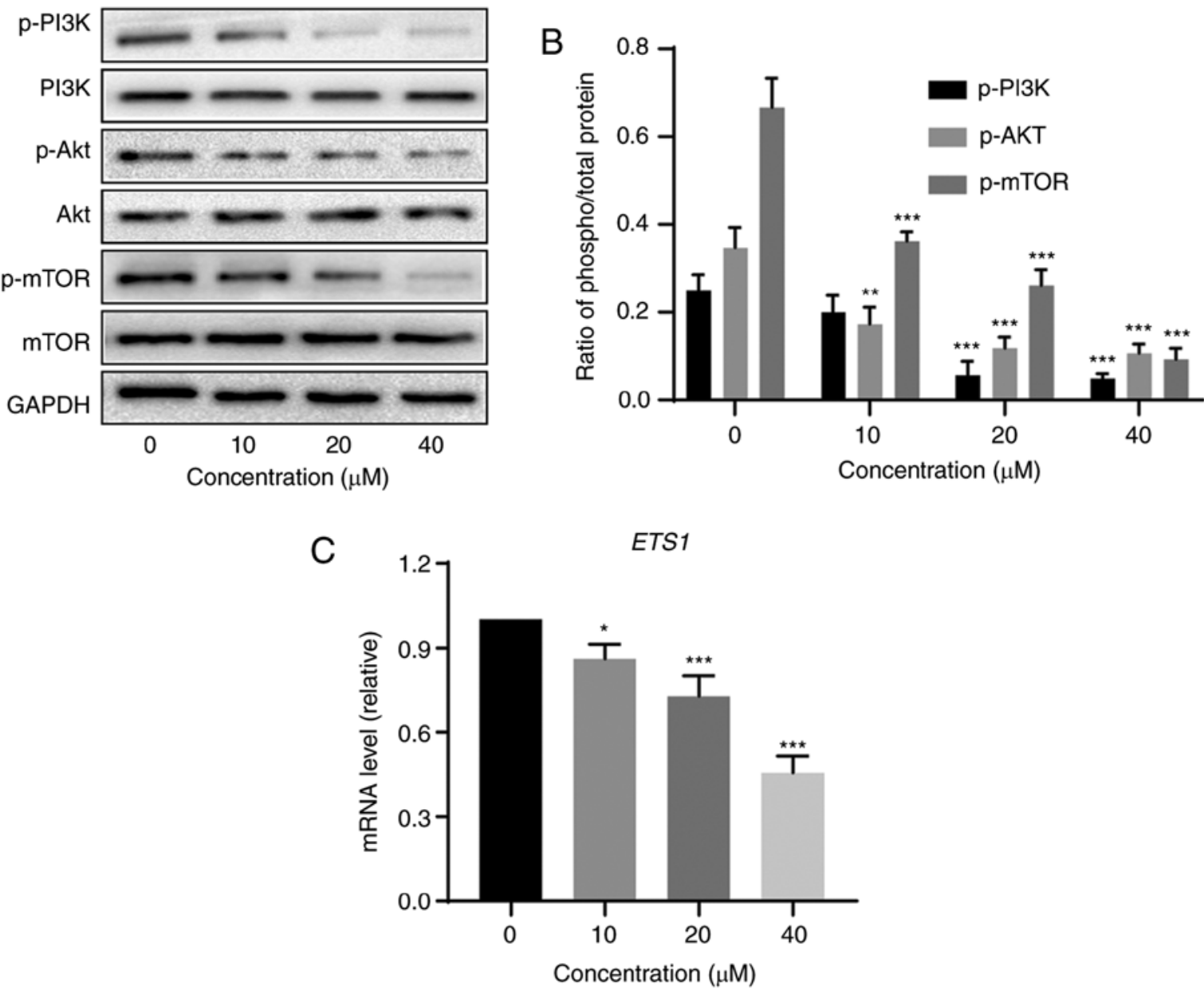

Figure 4. Veratramine inhibits the PI3K/Akt/mTOR signaling pathway in HepG2 cells. (A) Western blot analysis of PI3K, Akt and mTOR protein expression levels in HepG2 cells treated with various concentrations of veratramine $(0,10,20$ and $40 \mu \mathrm{M})$ for $48 \mathrm{~h}$. (B) Quantitative data of p-PI3K, p-Akt, p-mTOR phospho/total proteins. (C) The qPCR analysis of ETS1 mRNA level in HepG2 cells treated with various concentrations of veratramine $(0,10,20$ and $40 \mu \mathrm{M}$ ) for $48 \mathrm{~h}$. The data are presented as the mean $\pm \mathrm{SD}$ of three independent experiments. ${ }^{*} \mathrm{P}<0.05,{ }^{* * *} \mathrm{P}<0.01,{ }^{* * * *} \mathrm{P}<0.001$ vs. the control group. ETS1, ETS proto-oncogene 1 , transcription factor.

Bcl-2 family. Among them, $B C L 2$ is an anti-apoptotic gene, whereas $B A X$ is a pro-apoptotic gene. Studies have indicated that the ratio of $B C L 2$ to $B A X$ is considered to be a decisive factor as to whether a cell will commit to apoptosis. Therefore, in order to further verify whether veratramine can induce apoptosis, the mRNA expression levels of $B C L 2$ and $B A X$ were detected using RT-qPCR. RT-qPCR results revealed that veratramine significantly upregulated the expression level of $B A X$ and downregulated the expression level of $B C L 2$, which further demonstrated that veratramine induced apoptosis in HepG2 cells (Fig. 2C and D).

Veratramine induces autophagy in Hep 62 cells. To explore the mechanism of action behind the antitumor activity, the effect of veratramine on autophagy in HepG2 cells was investigated. AO staining was performed to label autophagosomes. Flow cytometric results revealed that the number of autophagic cells $\left(\mathrm{AO}^{+}\right)$increased with increasing veratramine concentrations. After $48 \mathrm{~h}$ of veratramine treatment $(40 \mu \mathrm{M})$, the proportion of autophagic cells reached $45.3 \%$ (Fig. 3A and B). Moreover, fluorescence microscopy images further confirmed that veratramine induced autophagy in HepG2 cells (Fig. 3C). Autophagy is strictly regulated by gene expression, with Beclin-1 and
LC3 proteins being markers of autophagy $(41,42)$. During autophagy, LC3-I is converted to LC3-II and then localized to the autophagosome membrane (41). The expression levels of LC3-II are positively correlated with the degree of autophagy, and the ratio of LC3-II/I can be used to estimate the level of autophagy $(41,42)$. In the present study, western blot analysis revealed that Beclin-1 and LC3-II proteins were significantly upregulated, while LC3-I protein was significantly downregulated, in the veratramine-treated cells. The Beclin-1 protein expression levels and the ratio of LC3-II/I were the highest in the high concentration group $(40 \mu \mathrm{M})$ (Fig. 3D-F). Overall, the aforementioned results demonstrated that veratramine treatment could induce autophagy in HepG2 cells.

Veratramine induces autophagy by inhibiting the PI3K/Akt/mTOR signaling pathway. The PI3K/Akt/mTOR signaling pathway is an intracellular signaling pathway that plays an important role in the pathogenesis of cancer (30). As a downstream protein of PI3K and Akt, phosphorylated mTOR protein promotes cancer cell proliferation and angiogenesis $(28,29)$. A previous study has demonstrated that the $\mathrm{PI} 3 \mathrm{~K} / \mathrm{Akt} / \mathrm{mTOR}$ pathway is associated with autophagy (43). Therefore, it was hypothesized that veratramine may inhibit 
A

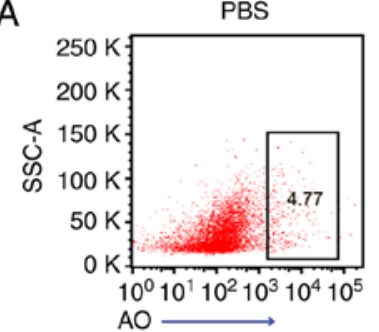

Veratramine

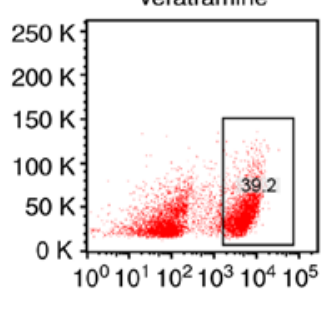

Veratramine+3-MA

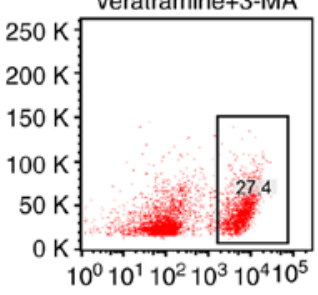

$\mathrm{B}$

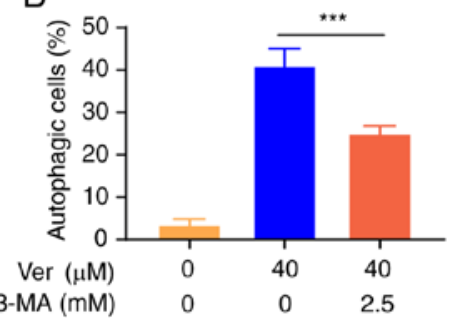

C

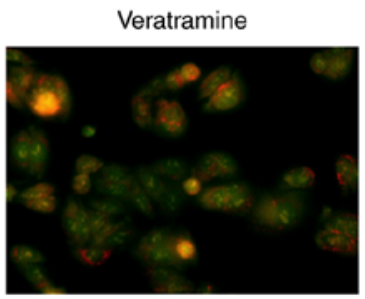

Veratramine+3-MA

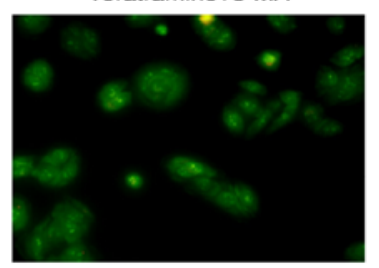

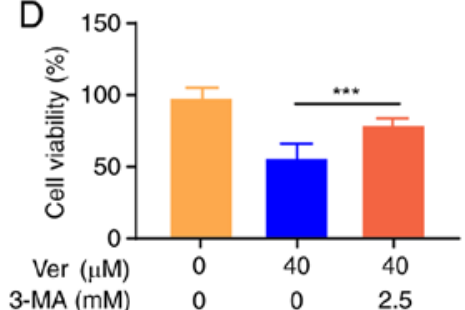

3-MA (mM) $0 \quad 02.5$
E

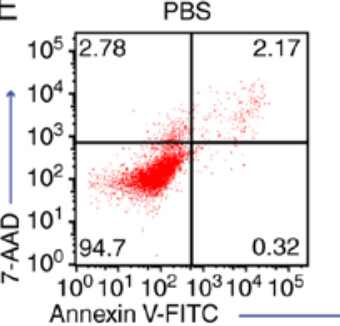

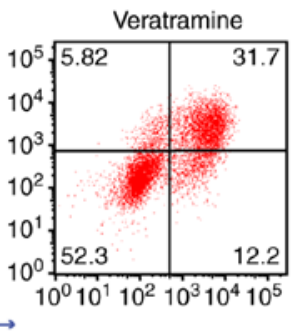

Veratramine+3-MA

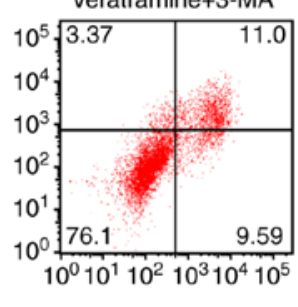

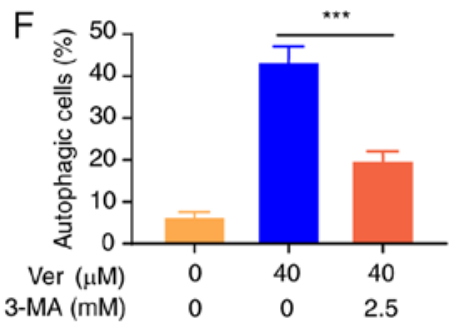

Figure 5. Autophagy inhibitor 3-MA inhibits veratramine-induced apoptosis in HepG2 cells. (A) Flow cytometric analysis of autophagic vacuoles (AO $\left.{ }^{+}\right)$in HepG2 cells treated with PBS control, veratramine $(40 \mu \mathrm{M})$ or veratramine $(40 \mu \mathrm{M})$ plus 3 -MA $(2.5 \mu \mathrm{M})$ for 48 h. (B) Percentages of the autophagic cells. (C) Representative fluorescence microscopy images of autophagic vacuoles (red) in HepG2 cells treated with veratramine in the presence or absence of 3-MA. (D) The cell viability after different treatment. (E) Flow cytometric analysis of apoptotic cells in HepG2 cells after treatment. (F) Quantitative analysis of apoptotic cell numbers (Annexin $\mathrm{V}^{+}$). The data are presented as the mean $\pm \mathrm{SD}$ of three independent experiments $\left({ }^{* * *} \mathrm{P}<0.001\right)$. 3-MA, 3-methyladenine.

the PI3K/Akt/mTOR pathway to promote autophagic cell death. In order to verify this hypothesis, the phosphorylation levels of PI3K, Akt and mTOR proteins were assessed using western blotting. The results revealed that the levels of p-PI3K, p-Akt and p-mTOR proteins were significantly lower in veratramine-treated cells than in the control cells, and the changes were achieved in a dose-dependent manner (Fig. 4A and B). Moreover, a previous study demonstrated that veratramine modulated $A P$-1-dependent gene transcription by directly binding to programmable DNA (35). ETS proto-oncogene 1, transcription factor (ETS1), an AP-1 downstream gene (35), has been revealed to be predominantly expressed in various tumor subtypes and can mediate activation of the PI3K/Akt/mTOR pathway $(44,45)$. As anticipated, the mRNA level of ETS1 was significantly downregulated in veratramine-treated HepG2 cells (Fig. 4C). Based on these results, it was concluded that veratramine induced autophagy by blocking the PI3K/Akt/mTOR signaling pathway in HepG2 cells.

Veratramine induces autophagy-mediated apoptosis in HepG2 cells. To evaluate the effect of autophagy on veratramine-induced cell death, autophagy inhibitor 3-MA was used and the proportion of apoptotic cells and the cell viability was examined. Cells were co-incubated with veratramine $(40 \mu \mathrm{M})$ for $48 \mathrm{~h}$ in the presence or absence of 3-MA (2.5 mM). As revealed in Fig. 5A and $\mathrm{B}$, after the addition of 3-MA, the number of autophagosomes was significantly decreased, demonstrating that autophagy induced by veratramine was significantly inhibited. Fluorescence microscopy results further confirmed that autophagic vacuoles were reduced after 3-MA co-treatment (Fig. 5C). Moreover, cell viability in cells treated with a combination of veratramine and 3-MA, was higher than that in cells treated with veratramine alone, which indicated that 3-MA attenuated the cytotoxicity of veratramine on HepG2 cells (Fig. 5D). Similarly, the proportion of apoptotic cells in the co-treatment group was lower compared with that of the veratramine alone treatment group (Fig. 5E and F). Collectively, these results indicated that veratramine induced-autophagy promoted apoptosis in HepG2 cells.

Veratramine suppresses liver cancer xenografted tumor growth with a low systemic toxicity. Based on the findings of the present in vitro experiments, the antitumor effect of veratramine was further verified in the HepG2 subcutaneous xenograft mouse model. No mice died or acted abnormally before the end of the animal experiment. By monitoring the tumor volume, it was observed that veratramine treatment significantly inhibited tumor growth compared with the PBS 
A

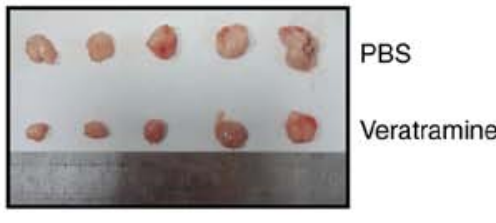

D

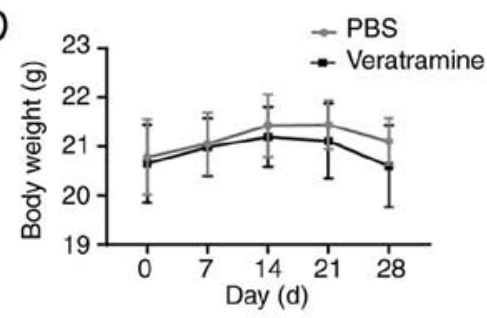

B

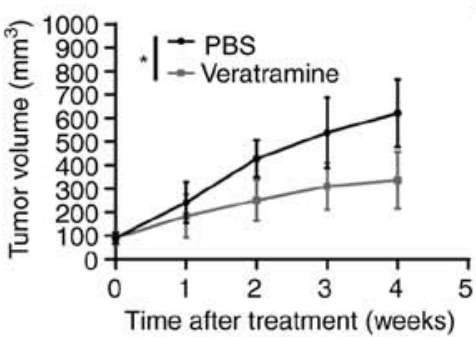

E

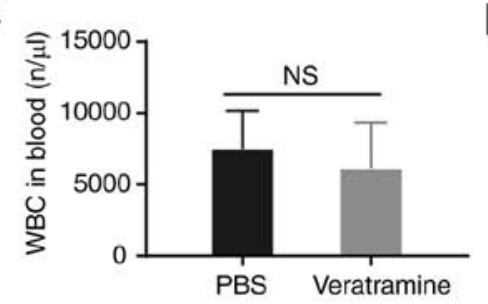

C

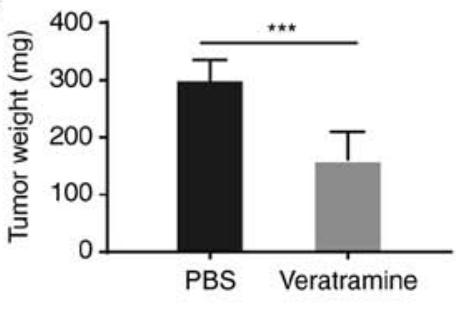

$\mathrm{F}$

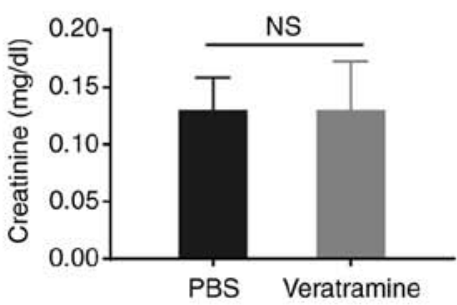

$\mathrm{G}$

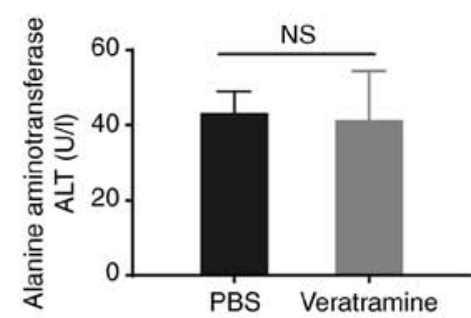

$\mathrm{H}$

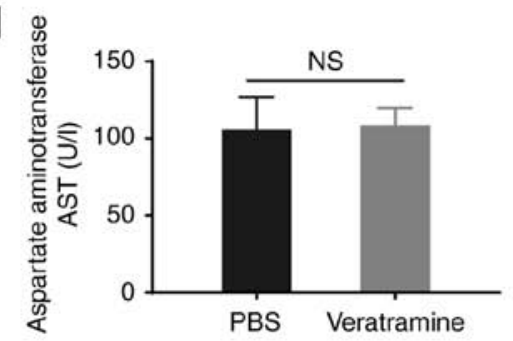

I
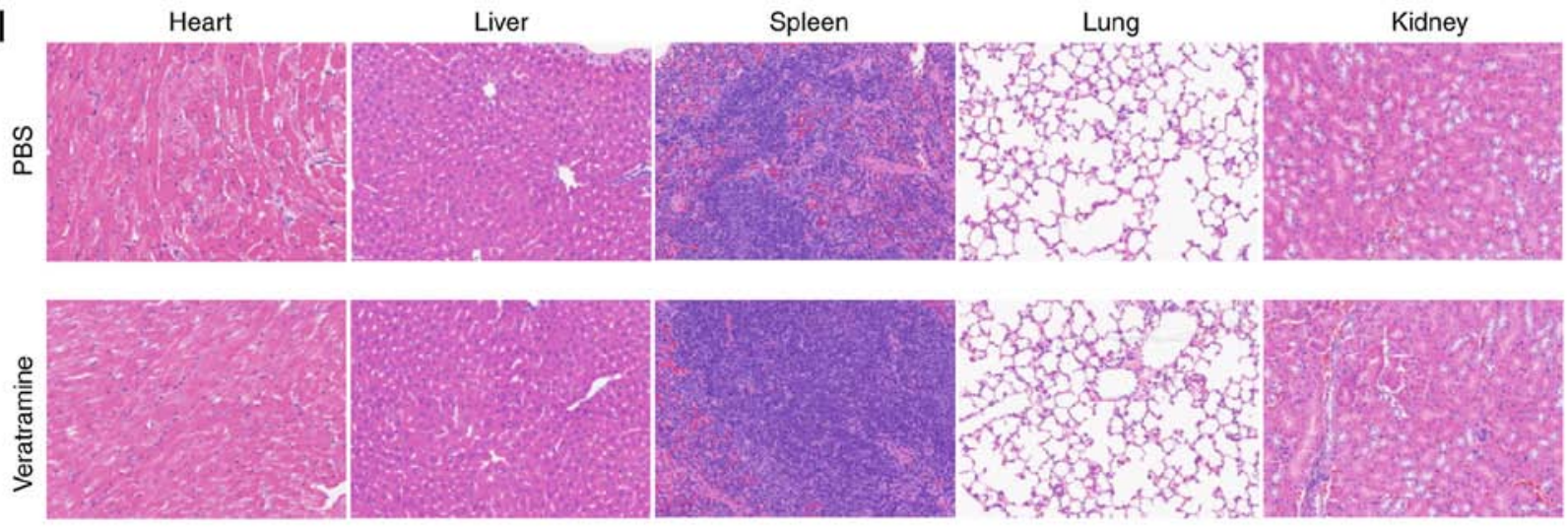

Figure 6. Veratramine suppresses tumor growth with low systemic toxicity in subcutaneous xerographic tumor-loaded nude mice. (A) Images of the tumors 3 days after the last treatment with PBS or veratramine. (B) Tumor volume curves. (C) Tumor weight. (D) Mice weight curves. (E) The number of WBCs in the blood. (F) Serum levels of creatinine. (G) Serum levels of ALT. (H) Serum levels of AST. (I) Representative H\&E staining images of tissue sections of the heart, liver, spleen and kidney collected from mice at the end of treatment. Data are presented as the mean $\pm \mathrm{SD}\left(\mathrm{n}=5\right.$, $\left.{ }^{*} \mathrm{P}<0.05,{ }^{* * *} \mathrm{P}<0.001\right)$. WBCs, white blood cells; ALT, alanine transferase; AST, alanine aminotransferase; NS, no significance.

control (Fig. 6A-B). Consistent with the tumor volume, tumor weight in the veratramine group was significantly lower than the tumor weight in the PBS group (Fig. 6C). In addition, the systemic toxicity of veratramine was also evaluated in vivo. The weight of the mice in the veratramine-treated group was not significantly different from that in the PBS group (Fig. 6D). Furthermore, veratramine treatment had no significant effect on the number of blood cells as well as the liver and kidney function compared with the PBS control (Fig. 6E-H). Moreover, the results of $\mathrm{H} \& \mathrm{E}$ staining revealed that no obvious tissue damage, such as inflammation (infiltration of inflammatory cells) and/or necrosis (tissue structural destruction), was observed in tissue sections of the heart, liver, spleen, lung and kidney (Fig. 6I), which indicated that veratramine had no obvious organ toxicity. Collectively, these results demonstrated that veratramine not only suppressed tumor growth in vivo, but also had no obvious systemic toxicity.

\section{Discussion}

Natural products are invaluable as resources for the discovery and development of various drugs, especially antitumor 
drugs (46-48). Numerous chemotherapeutic drugs derived from natural products, such as taxol and hydroxycamptothecin, have exhibited favorable clinical efficacy $(47,48)$. Veratramine, a steroidal alkaloid isolated from plants of the lily family, has been reported to possess multiple pharmacological activities, such as lowering blood pressure and acting as an antithrombotic agent $(49,50)$. Recent studies demonstrated that veratramine may have antitumor activities (34). Liver cancer is a malignant tumor that is insensitive to existing drug treatments (4), thus, it is extremely urgent to find a safe and effective drug to treat liver cancer. In the present study, veratramine was revealed to significantly inhibit cell proliferation, invasion and metastasis in HepG2 cells, and the $\mathrm{IC}_{50}$ value of veratramine on HepG2 cells at $48 \mathrm{~h}$ was only $19.81 \mu \mathrm{M}$, which demonstrated that veratramine had a strong antitumor effect. Notably, it was also observed that veratramine significantly inhibited tumor growth in a liver cancer subcutaneous xenograft model. In addition, common toxic side effects associated with chemotherapeutic drugs were also evaluated. It was revealed that veratramine had no significant effect on the number of blood cells, weight, and liver/kidney function in mice. Based on these findings, it was inferred that veratramine had characteristics such as a high efficiency and low systemic toxicity and was a promising antitumor agent.

Autophagy is a cellular metabolic process possessed by eukaryotes in which cytoplasmic cargo is delivered to lysosomes and intracellular components are degraded and recycled (10). Autophagy often occurs at low levels in cells and is rapidly upregulated when cells are under stress (8). Evidence suggests that induction of autophagic cell death is a promising strategy for the treatment of cancer (51). In the present study, it was revealed that veratramine could concurrently induce autophagy and apoptosis in HepG2 cells. To confirm the relationship between autophagy and apoptosis in veratramine-induced HepG2 cell death, 3-MA was used to inhibit autophagy. Notably, after autophagy was inhibited, the proportion of apoptotic cells was reduced, and cell viability was also reduced, which indicated that veratramine induced autophagy-mediated apoptosis in HepG2 cells.

mTOR kinase can act directly on autophagy-related proteins to regulate the formation of autophagosomes (52). The PI3/Akt/mTOR signaling pathway is one of the main pathways involved in autophagy, in which activated PI3K promotes the phosphorylation of Akt, leading to phosphorylation of mTOR, which inhibits autophagy $(30,43)$. In this present study, the data demonstrated that veratramine downregulated the phosphorylation levels of PI3K, AKT and mTOR in a concentration-dependent manner. Furthermore, ETS1, an $A P$-1-dependent gene, was also downregulated, consistent with a previous study (35). Previous studies have demonstrated that ETS1 mediates activation of the PI3K/Akt/mTOR pathway $(44,45)$. Therefore, it was concluded that veratramine inhibited the PI3K/AKT/mTOR signaling pathway by targeting ETS1, thereby inducing autophagic cell death in HepG2 cells. However, a limitation of the present study, is that whether the $A P-1$ gene itself and $A P-1$-dependent genes are involved in the antitumor effect of veratramine has not been explored.

In conclusion, the present data demonstrated that veratramine inhibited human liver cancer HepG2 cell proliferation, invasion and infiltration in vitro, and veratramine could also significantly suppress tumor growth with a low systemic toxicity in vivo. In addition, veratramine induced autophagy-mediated apoptosis in HepG2 cells by blocking the activation of the PI3K/Akt/mTOR signaling pathway. Therefore, the present study suggested that veratramine may be a potential agent for the treatment of liver cancer.

\section{Acknowledgments}

Not applicable.

\section{Funding}

The present study was supported by the National Natural Science Foundation of China (grant no. 81241102) and the fund of the Traditional Chinese Medicine Scientific Research Project of Anhui Provincial Health Department (2012zy59).

\section{Availability of data and materials}

All data generated or analyzed during this study are included in this published article.

\section{Authors' contributions}

LY and YX participated in its design and performed experiments, analyzed the data and wrote the draft manuscript. PX, WZ and YG interpreted the results, performed the animal experiments and reviewed the manuscript. FX and ZJ conceived the study, performed data analysis and manuscript editing and improvement. All authors read and approved the manuscript and agree to be accountable for all aspects of the research in ensuring that the accuracy or integrity of any part of the work are appropriately investigated and resolved.

\section{Ethics approval and consent to participate}

The animal experiments were approved by the Animal Care and Use Committee of Yijishan Hospital of Wannan Medical College.

\section{Patient consent for publication}

Not applicable.

\section{Competing interests}

The authors declare that they have no competing interests.

\section{References}

1. Siegel RL, Miller KD and Jemal A: Cancer statistics, 2019. CA Cancer J Clin 69: 7-34, 2019.

2. Kudo M: Systemic therapy for hepatocellular carcinoma: Latest advances. Cancers (Basel) 10: E412, 2018.

3. Li D, Sedano S, Allen R, Gong J, Cho M and Sharma S: Current treatment landscape for advanced hepatocellular carcinoma: Patient outcomes and the impact on quality of life. Cancers (Basel) 11: E841, 2019.

4. Colagrande S, Inghilesi AL, Aburas S, Taliani GG, Nardi C and Marra F: Challenges of advanced hepatocellular carcinoma. World J Gastroenterol 22: 7645-7659, 2016. 
5. Ikeda K: Recent advances in medical management of hepatocellular carcinoma. Hepatol Res 49: 14-32, 2019.

6. Kawaguchi T, Nakano D, Okamura S, Shimose S, Hayakawa M, Niizeki T, Koga $\mathrm{H}$ and Torimura T: Spontaneous regression of hepatocellular carcinoma with reduction in angiogenesis-related cytokines after treatment with sodium-glucose cotransporter 2 inhibitor in a cirrhotic patient with diabetes mellitus. Hepatol Res 49: 479-486, 2019.

7. Shimose S, Tanaka M, Iwamoto H, Niizeki T, Shirono T, Aino H, Noda Y, Kamachi N, Okamura S, Nakano M, et al: Prognostic impact of transcatheter arterial chemoembolization (TACE) combined with radiofrequency ablation in patients with unresectable hepatocellular carcinoma: Comparison with TACE alone using decision-tree analysis after propensity score matching. Hepatol Res 49: 919-928, 2019.

8. Doherty $\mathrm{J}$ and Baehrecke EH: Life, death and autophagy. Nat Cell Biol 20: 1110-1117, 2018.

9. Dikic I: Proteasomal and autophagic degradation systems. Annu Rev Biochem 86: 193-224, 2017.

10. Mizushima N and Komatsu M: Autophagy: Renovation of cells and tissues. Cell 147: 728-741, 2011.

11. Levine B and Kroemer G: Autophagy in the pathogenesis of disease. Cell 132: 27-42, 2008.

12. Retnakumar SV and Muller S: Pharmacological autophagy regulators as therapeutic agents for inflammatory bowel diseases Trends Mol Med 25: 516-537, 2019.

13. Long $\mathrm{M}$ and McWilliams TG: Monitoring autophagy in cancer: From bench to bedside. Semin Cancer Biol: Jul 15, 2019 (Epub ahead of print)

14. Yazdani HO, Huang H and Tsung A: Autophagy: Dual response in the development of hepatocellular carcinoma. Cells 8: E91, 2019.

15. Li YJ, Lei YH, Yao N, Wang CR, Hu N, Ye WC, Zhang DM and Chen ZS: Autophagy and multidrug resistance in cancer. Chin J Cancer 36: 52, 2017

16. Elgendy M, Ciro M, Abdel-Aziz AK, Belmonte G, Dal Zuffo R, Mercurio C, Miracco C, Lanfrancone L, Foiani M and Minucci S: Beclin 1 restrains tumorigenesis through Mcl-1 destabilization in an autophagy-independent reciprocal manner. Nat Commun 5: 5637,2014

17. Edinger AL and Thompson CB: Defective autophagy leads to cancer. Cancer Cell 4: 422-424, 2003.

18. Shimizu S, Yoshida T, Tsujioka M and Arakawa S: Autophagic cell death and cancer. Int J Mol Sci 15: 3145-3153, 2014.

19. Nikoletopoulou V, Markaki M, Palikaras K and Tavernarakis N: Crosstalk between apoptosis, necrosis and autophagy. Biochim Biophys Acta 1833: 3448-3459, 2013.

20. Gordy $\mathrm{C}$ and He YW: The crosstalk between autophagy and apoptosis: Where does this lead? Protein Cell 3: 17-27, 2012.

21. Lee EF, Smith NA, Soares da Costa TP, Meftahi N, Yao S, Harris TJ, Tran S, Pettikiriarachchi S, Perugini MA, Keizer DW, et al: Structural insights into BCL2 pro-survival protein interactions with the key autophagy regulator BECN1 following phosphorylation by STK4/MST1. Autophagy 15: 785-795, 2019

22. Levine B, Sinha SC and Kroemer G: Bcl-2 family members: Dual regulators of apoptosis and autophagy. Autophagy 4: 600-606, 2008

23. Wu H, Che X, Zheng Q, Wu A, Pan K, Shao A, Wu Q, Zhang J and Hong Y: Caspases: A molecular switch node in the crosstalk between autophagy and apoptosis. Int J Biol Sci 10: 1072-1083, 2014.

24. Hou W, Han J, Lu C, Goldstein LA and Rabinowich H: Autophagic degradation of active caspase-8: A crosstalk mechanism between autophagy and apoptosis. Autophagy 6: 891-900, 2010.

25. Djavaheri-Mergny M, Maiuri MC and Kroemer G: Cross talk between apoptosis and autophagy by caspase-mediated cleavage of Beclin 1. Oncogene 29: 1717-1719, 2010.

26. Salminen A, Kaarniranta K and Kauppinen A: Beclin 1 interactome controls the crosstalk between apoptosis, autophagy and inflammasome activation: Impact on the aging process. Ageing Res Rev 12: 520-534, 2013.

27. Qu X, Zou Z, Sun Q, Luby-Phelps K, Cheng P, Hogan RN, Gilpin C and Levine B: Autophagy gene-dependent clearance of apoptotic cells during embryonic development. Cell 128 : 931-946, 2007.

28. Paquette M, El-Houjeiri L and Pause A: mTOR pathways in cancer and autophagy. Cancers (Basel) 10: E18, 2018.

29. Boutouja F, Stiehm CM and Platta HW: mTOR: A cellular regulator interface in health and disease. Cells 8: E18, 2019.

30. Alzahrani AS: PI3K/Akt/mTOR inhibitors in cancer: At the bench and bedside. Semin Cancer Biol 59: 125-132, 2019.
31. Wagle N, Grabiner BC, Van Allen EM, Hodis E, Jacobus S, Supko JG, Stewart M, Choueiri TM, Gandhi L, Cleary JM, et al: Activating mTOR mutations in a patient with an extraordinary response on a phase I trial of everolimus and pazopanib. Cancer Discov 4: 546-553, 2014.

32. Jovanović B, Mayer IA, Mayer EL, Abramson VG, Bardia A, Sanders ME, Kuba MG, Estrada MV, Beeler JS, Shaver TM, et al: A randomized phase II neoadjuvant study of cisplatin, paclitaxel with or without everolimus in patients with stage II/III triple-negative breast cancer (TNBC): Responses and long-term outcome correlated with increased frequency of DNA damage response gene mutations, TNBC subtype, AR status, and Ki67. Clin Cancer Res 23: 4035-4045, 2017.

33. Li J, Kim SG and Blenis J: Rapamycin: One drug, many effects. Cell Metab 19: 373-379, 2014.

34. Tang J, Li HL, Shen YH, Jin HZ, Yan SK, Liu RH and Zhang WD: Antitumor activity of extracts and compounds from the rhizomes of veratrum dahuricum. Phytother Res 22: 1093-1096, 2008.

35. Bai F, Liu K, Li H, Wang J, Zhu J, Hao P, Zhu L, Zhang S, Shan L, Ma W, et al: Veratramine modulates AP-1-dependent gene transcription by directly binding to programmable DNA. Nucleic Acids Res 46: 546-557, 2018.

36. Livak KJ and Schmittgen TD: Analysis of relative gene expression data using real-time quantitative PCR and the 2(-Delta Delta C(T)) method. Methods 25: 402-408, 2001.

37. Tang J, Li HL, Shen YH, Jin HZ, Yan SK, Liu XH, Zeng HW, Liu RH, Tan YX and Zhang WD: Antitumor and antiplatelet activity of alkaloids from veratrum dahuricum. Phytother Res 24: 821-826, 2010.

38. Cong Y, Guo J, Tang Z, Lin S, Zhang Q, Li J and Cai Z Metabolism study of veratramine associated with neurotoxicity by using HPLC-MSn. J Chromatogr Sci 53: 1092-1099, 2015.

39. Lyu C, Zhang Y, Zhou W, Zhang S, Kou F, Wei H, Zhang N and Zuo Z: Gender-dependent pharmacokinetics of veratramine in rats: In vivo and in vitro evidence. AAPS J 18: 432-444, 2016.

40. Gross A, McDonnell JM and Korsmeyer SJ: BCL-2 family members and the mitochondria in apoptosis. Genes Dev 13: 1899-1911, 1999.

41. Yorimitsu T and Klionsky DJ: Autophagy: Molecular machinery for self-eating. Cell Death Differ 12 (Suppl 2): S1542-S1552, 2005.

42. Cao Y and Klionsky DJ: Physiological functions of Atg6/Beclin 1: A unique autophagy-related protein. Cell Res 17: 839-849, 2007.

43. Porta C, Paglino C and Mosca A: Targeting PI3K/Akt/mTOR signaling in cancer. Front Oncol 4: 64, 2014.

44. Xu S, Ge J, Zhang Z and Zhou W: MiR-129 inhibits cell proliferation and metastasis by targeting ETS1 via PI3K/AKT/mTOR pathway in prostate cancer. Biomed Pharmacother 96: 634-641, 2017.

45. Meng S, Jian Z, Yan X, Li J and Zhang R: LncRNA SNHG6 inhibits cell proliferation and metastasis by targeting ETS1 via the PI3K/AKT/mTOR pathway in colorectal cancer. Mol Med Rep 20: 2541-2548, 2019.

46. Tu Y, Zhu S, Wang J, Burstein E and Jia D: Natural compounds in the chemoprevention of alcoholic liver disease. Phytother Res 33 2192-2212, 2019.

47. Annovazzi L, Caldera V, Mellai M, Riganti C, Battaglia L, Chirio D, Melcarne A and Schiffer D: The DNA damage/repair cascade in glioblastoma cell lines after chemotherapeutic agent treatment. Int J Oncol 46: 2299-2308, 2015.

48. Dotan E, Cohen SJ, Starodub AN, Lieu CH, Messersmith WA, Simpson PS, Guarino MJ, Marshall JL, Goldberg RM, Hecht JR, et al: Phase I/II trial of labetuzumab govitecan (anti-CEACAM5/SN-38 antibody-drug conjugate) in patients with refractory or relapsing metastatic colorectal cancer. J Clin Oncol 35: 3338-3346, 2017.

49. Li Q, Zhao YL, Long CB, Zhu PF, Liu YP and Luo XD: Seven new veratramine-type alkaloids with potent analgesic effect from Veratrum taliense. J Ethnopharmacol 244: 112137, 2019.

50. Chandler CM and McDougal OM: Medicinal history of North American. Veratrum. Phytochem Rev 13: 671-694, 2014.

51. Deng S, Shanmugam MK, Kumar AP, Yap CT, Sethi G and Bishayee A: Targeting autophagy using natural compounds for cancer prevention and therapy. Cancer 125: 1228-1246, 2019.

52. Jung CH, Ro SH, Cao J, Otto NM and Kim DH: mTOR regulation of autophagy. FEBS Lett 584: 1287-1295, 2010.

This work is licensed under a Creative Commons Attribution-NonCommercial-NoDerivatives 4.0 International (CC BY-NC-ND 4.0) License. 